\title{
Positive or close margins: reoperation rate and second conservative resection or total mastectomy?
}

This article was published in the following Dove Medical Press journal: Cancer Management and Research

\author{
Gilles Houvenaeghel' \\ Eric Lambaudie' \\ Marie Bannier ${ }^{2}$ \\ Sandrine Rua ${ }^{2}$ \\ Julien Barrou ${ }^{2}$ \\ Mellie Heinemann' \\ Max Buttarelli2 \\ Jeanne Thomassin Piana ${ }^{3}$ \\ Monique Cohen ${ }^{2}$ \\ 'Department of Surgical Oncology, \\ Paoli Calmettes Institute and CRCM, \\ CNRS, INSERM, Aix Marseille \\ Université, 13009 Marseille, France; \\ ${ }^{2}$ Department of Surgical Oncology, \\ Paoli Calmettes Institute, I 3009 \\ Marseille, France; ${ }^{3}$ Department of \\ Pathology, Paoli Calmettes Institute \\ and CRCM, CNRS, INSERM, I 3009 \\ Marseille, France
}

Correspondence: Gilles Houvenaeghel Department of Surgical Oncology, Paoli Calmettes Institute and CRCM, CNRS, INSERM, Aix Marseille Université, 232 Bd de Sainte Marguerite, 13009 Marseille, France

Tel +33491223532

Fax +33 491223613

Email houvenaeghelg@ipc.unicancer.fr
Introduction: Reoperation after breast-conserving surgery (BCS) could be proposed for positive or close margins. Reoperation type, re-excision or mastectomy, depends on several factors in relation to patient's and tumor's characteristics. We have analyzed our breast cancer (BC) database in order to determine second and third attempts for BCS and mastectomy rates, as well as associated factors for type of surgery.

Methods: All patients with BCS between 1995 and 2017 were included. Patient's characteristics, pathologic results, and treatments were analyzed. Reoperation rate, type of reoperation, second reoperation, and associated factors of reoperation, mastectomy, and third intervention were determined. Three periods were determined: P1-P3.

Results: We analyzed 10,761 patients: 1,161 with ductal carcinoma in situ (DCIS) and 9,600 with invasive BC. The reoperation rate was $41.4 \%$ for DCIS and $28.0 \%$ for invasive BC. Using multivariate analysis, we identified tumor size $>20 \mathrm{~mm}$ as being a risk factor for reoperation, whereas age $>50$ years, $\mathrm{P} 2-3$, and some localization decreased reoperation rates. For invasive $\mathrm{BC}$, age $>40$ years, triple-negative tumors, neoadjuvant chemotherapy, and noncentral tumors decreased reoperation rates and lobular tumor, multifocal tumors, lymphovascular invasion, DCIS component, and Her2-positive tumors increased reoperation rates. For patients requiring reoperation, re-excision was performed in $48.1 \%(1,523 / 3,168)$ and mastectomy was required after first re-excision in $13.46 \%$ (205/1,523). For DCIS, mastectomy rates were higher for grade 2 and tumor $\geq 20 \mathrm{~mm}$. For invasive BC, mastectomy rates were higher for lobular, multifocal, $\geq 20 \mathrm{~mm}$, Her2-positive tumors and diffuse positive margins and lower for age $>50$ years and during the last period. Even if interval time between surgery and adjuvant treatments was higher for patients with reoperation, survival rates were not different between patients with and without reoperation.

Conclusion: A decrease in reoperation and mastectomy rates had been reported with several associated factors. A third intervention with mastectomy was required in $13.5 \%$ of patients. This information should be done in case of reoperation.

Keywords: breast cancer, conservative surgery, mastectomy, reoperation, margins

\section{Introduction}

Reoperation after breast-conserving surgery (BCS) for breast cancer (BC) could be proposed for positive or close margins. Guidelines for reoperation have evolved during the past years: for invasive $\mathrm{BC}$, margins $\geq 5 \mathrm{~mm}$ and then $2 \mathrm{~mm}$ and "no ink on tumor" are required, and for ductal carcinoma in situ (DCIS), margins $\geq 2 \mathrm{~mm}$ are usually required. 
Reoperation type, re-excision or mastectomy, depends on several factors in relation to patient's and pathological tumor characteristics, mainly 1) patient's choice informed of third reoperation risk and no postmastectomy radiotherapy (PMRT) for patients without axillary lymph node macrometastases; 2) breast size, tumor size, and tumor localization; and 3 ) possibility of surgical resection (ie, oncoplasty).

Several studies have reported reoperation rate and type of reoperation after $\mathrm{BCS},{ }^{1-15}$ but only a few focused on predictive factors determining reoperation type. ${ }^{11,13,16}$ We have analyzed institutional BC database in order to determine rates and associated factors for reoperation, mastectomy, and third reoperation.

\section{Patients and methods}

All patients undergoing BCS in our institution, between January 1995 and December 2017, were included, except those with cT4 and inflammatory BC. A process is in place for institutional BC database to ensure data integrity, accuracy, and completeness. Patient's and clinical characteristics (cTN stage, age, breast tumor localization), pathologic results (tumor size, Scarff-Bloom-Richardson [SBR] grade, lymphovascular invasion, histological type, tumor focality, phenotype, in situ component, focal [ $<3$ fields at magnification 40], or diffuse positive margins), and treatment (adjuvant chemotherapy [AC] or neoadjuvant chemotherapy [NAC]) were analyzed. Three periods were determined: P1 (1995-2004) with $\geq 5 \mathrm{~mm}$ margins required, P2 (2005-2010) with $\geq 2 \mathrm{~mm}$ margins required, and P3 (after 2010) with negative margins with no ink on tumor.

Reoperation rate and type of reoperation rates (re-excision or mastectomy) and second reoperation after re-excision were analyzed. Factors associated with reoperation, mastectomy, and third intervention were determined. We analyzed interval times between first surgery and reoperation for DCIS, for invasive $\mathrm{BC}$ with distinction between patients with or without AC. Overall survival and disease-free survival were compared between patients with and without reoperation.

BCS was undertaken with systematic resection from the subcutaneous layer to major pectoralis muscle and cutaneous resection when tumor was near the skin or when cutaneous retraction was observed. The specimen was pinned on a plate, oriented, and lateral margins inked.

We performed literature review in order to discuss these results.

\section{Statistics}

Univariate comparisons were performed using chi-square test. To assess the independent prognostic effect of the variables, a multivariable analysis, using binary logistic regression, was performed. The multivariable model was fitted for those factors that were statistically significant in the univariate analyses. ORs and 95\% CIs were estimated for each variable compared to the reference group. Survivals results were compared using the log-rank test. A two-sided $P$-value of $<0.05$ was considered as statistically significant. Data analysis was performed using SPSS version 16.0 (SPSS Inc., Chicago, IL, USA).

\section{Results}

\section{Patients}

A total of 10,761 patients were included in the analysis: 1,161 DCIS and 9,600 invasive BC. The characteristics of patients are reported in Table 1.

\section{Reoperation}

Reoperation rate was 29.4\% (3,168/10,761: 95\% CI 28.5-30.3): 41.4\% $(481 / 1,161)$ and $28.0 \%(2,687 / 9,600)$ for DCIS and invasive BC, respectively. For the total cohort, all factors analyzed were significantly different for reoperation rate in the univariate analysis (Table 1). In the multivariate analysis for DCIS, tumor size $\geq 20 \mathrm{~mm}$ increased reoperation rate and age $>50$ years, $\mathrm{P} 2-3$ periods, and tumor localization (upper superior quadrant and external superior and inferior quadrants) decreased reoperation rates (Table 2). In multivariate analysis for invasive $\mathrm{BC}$, lobular tumor, bifocal or multifocal tumors, presence of lymphovascular peritumoral invasion (LVI), DCIS component, and Her2-positive tumors increased reoperation rates and age $>40$ years, triple-negative tumors, NAC, and noncentral tumors decreased reoperation rates (Table 3 ).

\section{Reoperation type}

For patients, who needed reoperation, a mastectomy was performed at first reoperation in 51.9\% $(1,645 / 3,168 ; 241 / 481$ : $50.1 \%$ for DCIS and 1,404/2,687: $52.2 \%$ for invasive BC) and mastectomy was required after first reoperation with re-excision in 13.46\% (205/1,523: 35 for DCIS and 170 for invasive $\mathrm{BC}$ ) with a total mastectomy rate of $58.4 \%$ (1,850/3,168; 276/481: $57.4 \%$ for DCIS and 1,574/2,687: $58.6 \%$ for invasive BC). For DCIS, the mastectomy rate was higher for grade 2 tumors and tumor sizes $\geq 20 \mathrm{~mm}$ (Table 4 ). For invasive $\mathrm{BC}$, the mastectomy rate was higher for lobular tumors, bifocal or multifocal tumors, tumor sizes $\geq 20 \mathrm{~mm}$, Her2-positive tumors, and diffuse positive margins and lower for age $>50$ years and patients treated during the last period (Table 5). 


\begin{tabular}{|c|c|c|c|c|c|c|c|c|c|c|c|}
\hline$\approx$ &  & $\begin{array}{l}\overline{0} \\
0 \\
\dot{0}\end{array}$ & 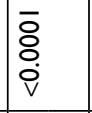 & \begin{tabular}{|l} 
\\
\\
$\dot{0}$ \\
$\dot{v}$
\end{tabular} & $\begin{array}{l}\bar{o} \\
\text { o } \\
\dot{v}\end{array}$ & $\begin{array}{l}\bar{\delta} \\
\text { o } \\
\dot{v}\end{array}$ & $\begin{array}{l}\bar{\delta} \\
\grave{i} \\
\dot{v}\end{array}$ & 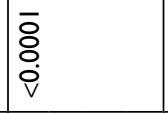 & $\begin{array}{l}\bar{\delta} \\
\grave{i} \\
\dot{i}\end{array}$ & $\begin{array}{l}\bar{\Xi} \\
\text { o } \\
\dot{v}\end{array}$ &  \\
\hline & ஃ & 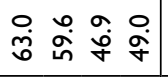 & 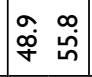 & 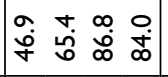 & 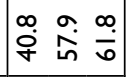 & 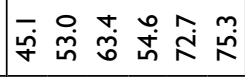 & 急 & 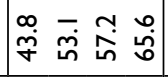 & 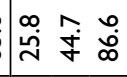 & 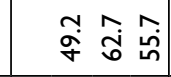 & à \\
\hline 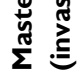 &  & 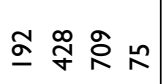 &  & $\underline{0} \underline{\underline{0}} \overline{\underline{m}} \boldsymbol{\infty}$ &  & 흐 & $\mid \begin{array}{ll} \\
\infty\end{array}$ & 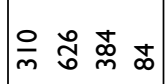 & œ 8 &  & 帒 \\
\hline$\approx$ & 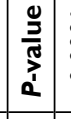 & $\begin{array}{l}\bar{o} \\
\text { o. } \\
\dot{0} \\
\end{array}$ & $\begin{array}{l}\bar{o} \\
\text { o } \\
\dot{0}\end{array}$ & \begin{tabular}{|l}
$\bar{Q}$ \\
\\
$\dot{0}$ \\
\end{tabular} & $\begin{array}{l}\overline{8} \\
\text { o } \\
\dot{0}\end{array}$ & $\begin{array}{l}\overline{8} \\
\text { o } \\
\dot{0} \\
\end{array}$ & $\begin{array}{l}\overline{8} \\
\text { ¿ } \\
\dot{0} \\
\end{array}$ & \begin{tabular}{|l}
$\overline{8}$ \\
o \\
v \\
\end{tabular} & $\begin{array}{l}\overline{8} \\
\text { i } \\
\text { v }\end{array}$ & $\begin{array}{l}\overline{8} \\
\text { ¿ } \\
\text { v }\end{array}$ & ס্் \\
\hline 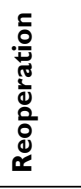 & $\circ \circ$ & $\widehat{\infty} \bar{m} \bar{m} \overline{\dot{d}}$ & 悹 & 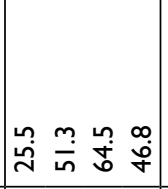 & $\stackrel{m}{m} \underset{\infty}{\infty} \stackrel{m}{=}$ & 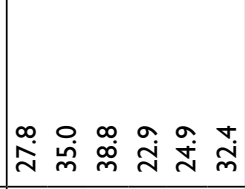 & 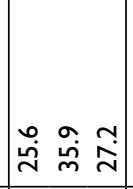 &  & مُ & 帒 & 文 \\
\hline 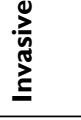 & $=1$ & 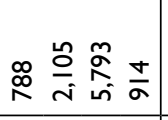 &  & 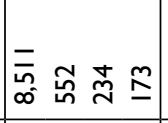 & 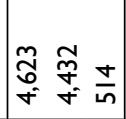 & 芯 & 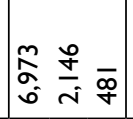 & 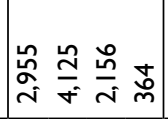 & 总言 & $\stackrel{\infty}{\stackrel{\infty}{N} \stackrel{n}{N}} \stackrel{0}{=}$ & 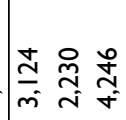 \\
\hline$\approx$ & 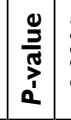 & $\begin{array}{l}\overline{0} \\
0 . \\
0\end{array}$ & & రั & & & & $\frac{m}{0}$ & סे & & ষ্ণ \\
\hline  & ○० & 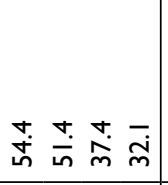 & & 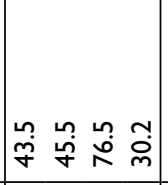 & & & & 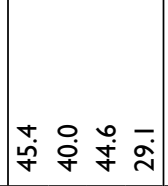 & $\hat{\sim}$ & &  \\
\hline Uू & $=1$ & 웃욧 n & & 架 นn & & & & |⿱ㅠㅇ \& & : & & $\stackrel{\infty}{\infty} \underset{\sim}{\sim} \stackrel{\alpha}{\sim}$ \\
\hline$\approx$ &  & $\begin{array}{l}\bar{o} \\
\text { o } \\
\dot{0}\end{array}$ & $\begin{array}{l}\bar{\delta} \\
\text { ò } \\
\dot{0} \\
\end{array}$ & $\begin{array}{l}\overline{8} \\
\text { ì } \\
\dot{0}\end{array}$ & $\begin{array}{l}\overline{8} \\
\text { ¿ } \\
\dot{v}\end{array}$ & $\begin{array}{l}\bar{\delta} \\
\text { ¿ } \\
\dot{v}\end{array}$ & $\begin{array}{l}\overline{8} \\
\text { ¿ } \\
\dot{v} \\
\end{array}$ & চे & $\begin{array}{l}\overline{8} \\
\dot{0} \\
\dot{v} \\
\end{array}$ &  & $\begin{array}{l}\overline{8} \\
\text { ¿ } \\
\dot{0} \\
\end{array}$ \\
\hline 实 & $\circ$ & 字家芯 & $\mid \begin{array}{ll}a & 0 \\
\dot{\sim} & \stackrel{0}{m} \\
\end{array}$ & 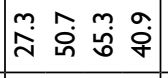 & $\stackrel{+}{\sim} \underset{\sim}{\stackrel{\infty}{m}} \stackrel{m}{=}$ & 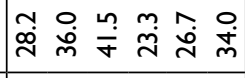 & वें &  & $\stackrel{0}{\circ}$ & $\mid \begin{array}{rr}\forall \\
\bar{\sigma}\end{array}$ &  \\
\hline  & $=$ & 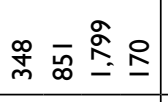 &  &  & 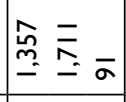 & $\stackrel{\hat{N}}{\underline{N}} \frac{o}{\sim} \stackrel{\infty}{=} \bar{i} \stackrel{\infty}{\infty} \stackrel{\infty}{\infty}$ & 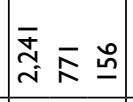 & $\frac{n}{n} \frac{8}{=} \frac{\infty}{0}$ & 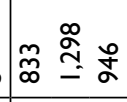 & 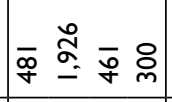 & 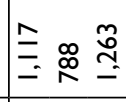 \\
\hline $\begin{array}{l}\bar{J} \\
\text { 。 } \\
\end{array}$ & $=$ & 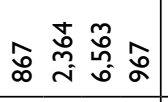 & 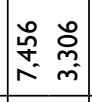 & 总 & 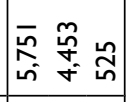 & 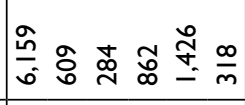 & 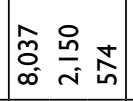 & 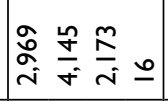 & 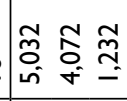 & 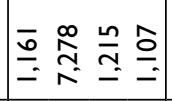 & 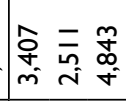 \\
\hline & & 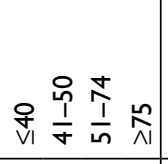 & 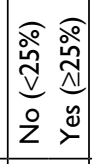 & 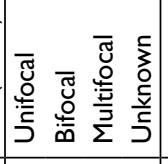 & 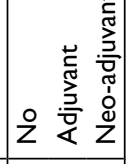 & 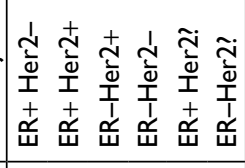 & 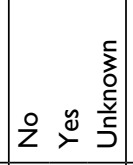 & 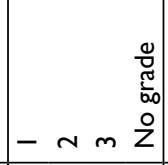 & 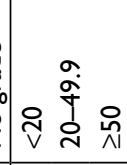 & 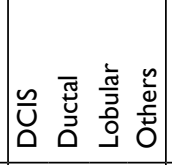 & $\mid \begin{array}{ll}0 & 0 \\
0 & 0 \\
0 & 0 \\
1 & 1\end{array}$ \\
\hline 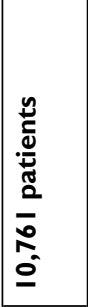 & & 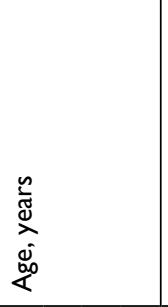 & 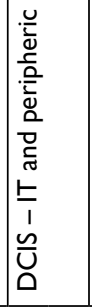 & 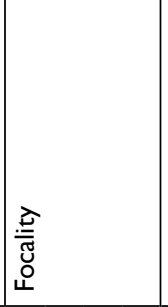 & 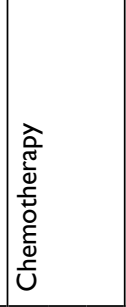 &  & $\sum$ & \begin{tabular}{|l} 
\\
$\stackrel{0}{\pi}$ \\
$\frac{\pi}{0}$ \\
\end{tabular} &  &  & \begin{tabular}{l}
$\frac{y}{0}$ \\
\hdashline$\frac{0}{0}$ \\
0 \\
0
\end{tabular} \\
\hline
\end{tabular}




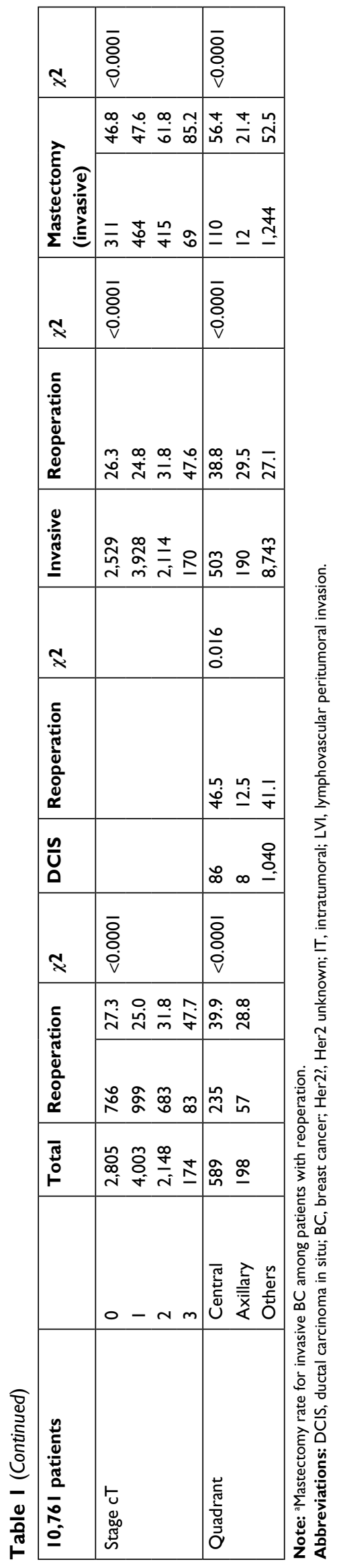

Among patients with re-excision, factors associated with reoperation with mastectomy in binary logistic regression were tumor size $\geq 20 \mathrm{~mm}$; multifocal tumors, which increased mastectomy rates; and age $>50$ years, which decreased mastectomy rates (Table 6).

\section{Interval time}

The median interval time between first surgery and reoperation for DCIS was 35 days (mean: $44.58,95 \%$ CI 41.3-47.8) and 78 days for patients with a second reoperation (mean 90.9, 95\% CI 75.9-105.8). Median interval time between first operation and radiotherapy for patients with DCIS was 55 days (mean 63, 95\% CI 60.4-65.6) and for patients with re-excision $(\mathrm{n}=238)$ or no reoperation $(\mathrm{n}=562)$, it was 77 days (mean 89.8, 95\% CI 83.4-96.1) or 47 days (mean 51.7, 95\% CI 49.8-53.6), respectively.

The median interval time between first surgery and reoperation (first reoperation) for invasive BC was 29 days (mean 47.7, 95\% CI 39.6-55.8) and 123 days for patients with a second reoperation (mean: 133, 95\% CI 121-145). When AC was administered, reoperation was performed before or after chemotherapy. The median interval time between first surgery and reoperation for invasive BC was 28 days (mean 33, 95\% CI 31.8-34.1) for patients without any treatment before reoperation (AC after reoperation or patients with previous NAC or patients without AC before reoperation) and 173.5 days (mean 179, 95\% CI 174-184) for patients with AC before reoperation.

The median time interval between first operation and radiotherapy for patients with invasive $\mathrm{BC}$ without $\mathrm{AC}$ was 73 days (mean 142 days, 95\% CI 14-270) and 46 days for patients with or without reoperation (mean 47.3 days, $95 \%$ CI 46-48), respectively.

For patients with invasive $\mathrm{BC}$ and $\mathrm{AC}$, the median interval time between first operation and $\mathrm{AC}$ was 42 days (mean 47.3 days, $95 \%$ CI 45.8-48.9) and 34 days (mean 36.9 days, $95 \%$ CI 36-38) for patients with reoperation and without reoperation, respectively, and the median interval time between first operation and radiotherapy was 188 days (mean 186.5 days, $95 \% \mathrm{CI}$ 184-189) and 167 days (mean 158 days, 95\% CI 156-160) for patients with reoperation and without reoperation, respectively.

\section{Survival}

The median follow-up was 53.2 months (95\% CI 56.7-89.0 months). Overall survival and disease-free survival were not different between patients with and without reoperation (log-rank test: 0.393 and 0.963 for invasive $\mathrm{BC}$ and 0.212 and 0.231 for DCIS). 
Table 2 Factors associated with reoperation for DCIS BC: multivariate analysis

\begin{tabular}{|c|c|c|c|c|c|}
\hline \multirow[t]{2}{*}{ Reoperation DCIS } & & \multirow[t]{2}{*}{$P$-value } & \multirow[t]{2}{*}{ OR } & \multicolumn{2}{|l|}{$95 \% \mathrm{Cl}$} \\
\hline & & & & Inferior & Superior \\
\hline Localization & $\begin{array}{l}\text { Central } \\
\text { Axillary } \\
\text { EIQ } \\
\text { IIQ } \\
\text { ESQ } \\
\text { ISQ } \\
\text { eEQ } \\
\text { elnfQ } \\
\text { elntQ } \\
\text { eSupQ }\end{array}$ & $\begin{array}{l}0.122 \\
0.052 \\
0.922 \\
0.036 \\
0.092 \\
0.572 \\
0.911 \\
0.740 \\
0.056\end{array}$ & $\begin{array}{l}1 \\
0.149 \\
0.481 \\
1.040 \\
0.531 \\
0.538 \\
1.233 \\
0.951 \\
1.150 \\
0.489\end{array}$ & $\begin{array}{l}0.013 \\
0.230 \\
0.474 \\
0.295 \\
0.261 \\
0.596 \\
0.393 \\
0.505 \\
0.235\end{array}$ & $\begin{array}{l}1.662 \\
1.008 \\
2.283 \\
0.958 \\
1.106 \\
2.554 \\
2.299 \\
2.620 \\
1.018 \\
\end{array}$ \\
\hline Age, years & $\begin{array}{l}\leq 40 \\
4 I-50 \\
5 I-74 \\
\geq 75\end{array}$ & $\begin{array}{l}0.961 \\
0.010 \\
0.054\end{array}$ & $\begin{array}{l}I \\
0.984 \\
0.464 \\
0.425\end{array}$ & $\begin{array}{l}0.522 \\
0.259 \\
0.177\end{array}$ & $\begin{array}{l}1.856 \\
0.831 \\
1.016\end{array}$ \\
\hline Periods & $\begin{array}{l}1995-2004 \\
2005-2010 \\
>2010\end{array}$ & $\begin{array}{l}0.019 \\
<0.0001\end{array}$ & $\begin{array}{l}I \\
0.601 \\
0.337 \\
\end{array}$ & $\begin{array}{l}0.392 \\
0.232 \\
\end{array}$ & $\begin{array}{l}0.920 \\
0.488\end{array}$ \\
\hline Grade & $\begin{array}{l}1 \\
2 \\
3 \\
\text { Unknown }\end{array}$ & $\begin{array}{l}0.347 \\
0.209 \\
0.028 \\
\end{array}$ & $\begin{array}{l}I \\
0.813 \\
0.756 \\
0.499 \\
\end{array}$ & $\begin{array}{l}0.529 \\
0.488 \\
0.268 \\
\end{array}$ & $\begin{array}{l}1.251 \\
1.170 \\
0.929 \\
\end{array}$ \\
\hline Size, $\mathrm{mm}$ & $\begin{array}{l}<20 \\
20-49.9 \\
\geq 50\end{array}$ & $\begin{array}{l}<0.0001 \\
<0.0001\end{array}$ & $\begin{array}{l}I \\
3.759 \\
17.76\end{array}$ & $\begin{array}{l}2.697 \\
11.18\end{array}$ & $\begin{array}{l}5.240 \\
28.21\end{array}$ \\
\hline
\end{tabular}

Abbreviations: DCIS, ductal carcinoma in situ; BC, breast cancer; EIQ, external inferior quadrants; IIQ, internal inferior quadrant; ESQ, external superior quadrant; ISQ, internal superior quadrant; eEQ, equatorial external quadrant; elnfQ, equatorial inferior quadrant; elntQ, equatorial internal quadrant; eSupQ, equatorial superior quadrant.

\section{Discussion}

In our study, the reoperation rate for DCIS was high (41.4\%) but lower during the two last periods (decrease of $24.6 \%$ ), and higher mastectomy rates (49.9\%) were reported for tumors $\geq 20$ and $<50 \mathrm{~mm}$. These results are concordant with literature results. ${ }^{17}$ Higher reoperation rates were also observed in the literature survey results for lobular carcinomas. The reoperation rate for invasive $\mathrm{BC}$ was $28.0 \%$, higher for young patients, triple-negative and Her2-positive tumors, lobular carcinomas, and multifocal tumors and lesser after NAC. Higher mastectomy rates for reoperation were also observed in our study mainly for these same factors. However, NAC was proposed for patients who need mastectomy before NAC or for patients with aggressive tumors (ie, SBR grade 3, Her2-positive, triple negative) and axillary involvement at ultrasonography and percutaneous biopsy.

In literature review, the mean reoperation rate (re-excision or mastectomy) was $27.49 \%$ (95\% CI $27.4-27.6$, range: $10.2 \%-34 \%$ ) among 402,357 patients with BC (DCIS or invasive) operation. ${ }^{1-15}$
Re-excision rates for BCS are extremely variable across the literature. This is mainly due to no clear consensus regarding the definition of a negative margin, different preoperative and intraoperative tumor localizing methods, differences in intraoperative imaging techniques, specimen inking by surgeons or pathologists, the use of shave margins, tumor vs lumpectomy size, oncoplastic resections, volume of breast surgery per year by surgeons, and surgeon threshold to offer re-excision vs mastectomy for positive margins..$^{13,18,19}$

When analysis of reoperation rates was performed according to three periods of treatment, we observed a decrease in reoperation rates among successive periods from $28.96 \%(4,594 / 15,861,95 \%$ CI $28.3-29.7)$ to $30.66 \%(79,708 / 259,943,95 \%$ CI $30.5-30.8)$ and $21.34 \%$ $(20,016 / 93,779,95 \%$ CI $21.1-21.6) \cdot)^{1,3-7,9-15}$ In the study reported by Morrow et al, ${ }^{9}$ surgery after initial lumpectomy declined by $16 \%(P<0.001)$ from 2013 to 2015 .

Reoperation rates were different for invasive $\mathrm{BC}$ and DCIS in literature reviews: $26.56 \%(86,797 / 326,828,95 \%$ CI 26.4-26.7, range: $10.4-30.5)$ and $33.1 \%(21,594 / 65,261$, 95\% CI 32.7-33.5, range: $8.97-41.4),{ }^{1-3,7,14}$ respectively. 
Table 3 Factors associated with reoperation for invasive BC: multivariate analysis

\begin{tabular}{|c|c|c|c|c|c|}
\hline \multirow[t]{2}{*}{ Reoperation invasive } & & \multirow[t]{2}{*}{$P$-value } & \multirow[t]{2}{*}{ OR } & \multicolumn{2}{|l|}{$95 \% \mathrm{Cl}$} \\
\hline & & & & Inferior & Superior \\
\hline Localization & $\begin{array}{l}\text { Central } \\
\text { Axillary } \\
\text { EIQ } \\
\text { IIQ } \\
\text { ESQ } \\
\text { ISQ } \\
\text { Inf M fold } \\
\text { eEQ } \\
\text { elnfQ } \\
\text { elntQ } \\
\text { eSupQ }\end{array}$ & $\begin{array}{l}0.020 \\
<0.000 \text { I } \\
<0.000 \text { I } \\
<0.000 \text { I } \\
<0.000 \text { I } \\
0.001 \\
<0.000 \text { I } \\
<0.000 \text { I } \\
<0.000 \text { I } \\
<0.0001\end{array}$ & $\begin{array}{l}1 \\
0.618 \\
0.582 \\
0.563 \\
0.527 \\
0.505 \\
0.087 \\
0.624 \\
0.431 \\
0.494 \\
0.531\end{array}$ & $\begin{array}{l}0.412 \\
0.445 \\
0.413 \\
0.422 \\
0.392 \\
0.020 \\
0.480 \\
0.310 \\
0.352 \\
0.412\end{array}$ & $\begin{array}{l}0.927 \\
0.762 \\
0.766 \\
0.659 \\
0.651 \\
0.383 \\
0.813 \\
0.601 \\
0.693 \\
0.684\end{array}$ \\
\hline Age, years & $\begin{array}{l}\leq 40 \\
4 I-50 \\
5 I-74 \\
\geq 75\end{array}$ & $\begin{array}{l}0.019 \\
<0.0001 \\
<0.0001\end{array}$ & $\begin{array}{l}I \\
0.786 \\
0.558 \\
0.276\end{array}$ & $\begin{array}{l}0.642 \\
0.463 \\
0.212\end{array}$ & $\begin{array}{l}0.961 \\
0.672 \\
0.359\end{array}$ \\
\hline Periods & $\begin{array}{l}1995-2004 \\
2005-2010 \\
>2010 \\
\end{array}$ & $\begin{array}{l}0.613 \\
0.863\end{array}$ & $\begin{array}{l}I \\
0.041 \\
0.013\end{array}$ & $\begin{array}{l}0.891 \\
0.876\end{array}$ & $\begin{array}{l}1.217 \\
1.170\end{array}$ \\
\hline NAC & NAC & 0.006 & 0.651 & 0.478 & 0.886 \\
\hline Histology & $\begin{array}{l}\text { Ductal } \\
\text { Lobular } \\
\text { Others }\end{array}$ & $\begin{array}{l}<0.0001 \\
0.819\end{array}$ & $\begin{array}{l}1 \\
0.960 \\
0.979 \\
\end{array}$ & $\begin{array}{l}1.678 \\
0.818\end{array}$ & $\begin{array}{l}2.290 \\
1.172\end{array}$ \\
\hline Focality & $\begin{array}{l}\text { Unifocal } \\
\text { Bifocal } \\
\text { Multifocal } \\
\text { Unknown }\end{array}$ & $\begin{array}{l}<0.0001 \\
<0.0001 \\
<0.0001\end{array}$ & $\begin{array}{l}1 \\
2.911 \\
4.717 \\
3.481\end{array}$ & $\begin{array}{l}2.398 \\
3.448 \\
2.214 \\
\end{array}$ & $\begin{array}{l}3.534 \\
6.454 \\
5.472 \\
\end{array}$ \\
\hline LVI & $\begin{array}{l}\text { No } \\
\text { Yes }\end{array}$ & 0.004 & $\begin{array}{l}1 \\
1.211\end{array}$ & 1.064 & 1.378 \\
\hline & Unknown & 0.025 & 1.383 & 1.042 & 1.836 \\
\hline DCIS component & DCIS - IT/peripheric & $<0.0001$ & 2.490 & 2.237 & 2.772 \\
\hline Grade & $\begin{array}{l}1 \\
2 \\
3 \\
\text { Unknown }\end{array}$ & $\begin{array}{l}0.954 \\
0.766 \\
0.474\end{array}$ & $\begin{array}{l}1 \\
0.004 \\
1.026 \\
1.152 \\
\end{array}$ & $\begin{array}{l}0.884 \\
0.867 \\
0.782 \\
\end{array}$ & $\begin{array}{l}1.140 \\
1.213 \\
1.698\end{array}$ \\
\hline Phenotype & $\begin{array}{l}\text { ER+ Her2- } \\
\text { ER+ Her2+ } \\
\text { ER- Her2+ } \\
\text { ER- Her2- } \\
\text { ER+ Her2? } \\
\text { ER- Her2? }\end{array}$ & $\begin{array}{l}0.037 \\
0.001 \\
0.041 \\
<0.0001 \\
0.302 \\
\end{array}$ & $\begin{array}{l}1 \\
1.252 \\
1.692 \\
0.802 \\
0.608 \\
0.848 \\
\end{array}$ & $\begin{array}{l}1.014 \\
1.235 \\
0.649 \\
0.504 \\
0.619 \\
\end{array}$ & $\begin{array}{l}1.545 \\
2.317 \\
0.991 \\
0.733 \\
1.160 \\
\end{array}$ \\
\hline Size invasive, $\mathbf{m m}$ & $\begin{array}{l}<20 \\
20-49.9 \\
\geq 50\end{array}$ & $\begin{array}{l}<0.0001 \\
<0.0001\end{array}$ & $\begin{array}{l}1 \\
1.434 \\
9.324\end{array}$ & $\begin{array}{l}1.280 \\
7.389\end{array}$ & $\begin{array}{l}1.607 \\
11.766\end{array}$ \\
\hline
\end{tabular}

Abbreviations: BC, breast cancer; EIQ, external inferior quadrant; IIQ, internal inferior quadrant; ESQ, external superior quadrant; ISQ, internal superior quadrant; Inf M fold, inferior mammary fold; eEQ, equatorial external quadrant; elnfQ, equatorial inferior quadrant; elntQ, equatorial internal quadrant; eSupQ, equatorial superior quadrant; NAC, neoadjuvant chemotherapy; LVI, lymphovascular peritumoral invasion; DCIS, ductal carcinoma in situ; IT, intratumoral.

However, reoperation rates differed between histological types of invasive BC: $26.98 \%$ for ductal invasive BC, $40.6 \%$ for lobular invasive $\mathrm{BC}$, and $21.83 \%$ for others invasive histological types. ${ }^{2}$

For DCIS, there was no statistically significant difference in locoregional recurrence (LRR) for patients with margins
$<2$ versus $\geq 2 \mathrm{~mm}$ who received radiotherapy (10-year LRR $4.8 \%$ vs $3.3 \%$, respectively; $P=0.72) .{ }^{20}$ One other large study evaluating the relationship between margin width and recurrence did not identify a significant association of recurrence with margin width of $\leq 2 \mathrm{~mm}$ compared with larger margins for patients receiving radiotherapy. ${ }^{21}$ However, Morrow et $\mathrm{al}^{22}$ 
Table 4 Factors associated with reoperation with mastectomy for DCIS BC: multivariate analysis

\begin{tabular}{|c|c|c|c|c|c|}
\hline \multirow{2}{*}{\multicolumn{2}{|c|}{$\begin{array}{l}\text { Mastectomy reoperation } \\
\text { DCIS }\end{array}$}} & \multirow{3}{*}{$\begin{array}{l}\boldsymbol{P} \text {-value } \\
\text { NS }\end{array}$} & \multirow{3}{*}{$\begin{array}{l}\text { OR } \\
1\end{array}$} & \multicolumn{2}{|l|}{$95 \% \mathrm{Cl}$} \\
\hline & & & & \multirow[t]{2}{*}{ Inferior } & \multirow[t]{2}{*}{ Superior } \\
\hline Localization & $\begin{array}{l}\text { Central } \\
\text { All others }\end{array}$ & & & & \\
\hline Age, years & $\begin{array}{l}\leq 40 \\
4 I-50 \\
5 I-74 \\
\geq 75\end{array}$ & $\begin{array}{l}0.854 \\
0.740 \\
0.939\end{array}$ & $\begin{array}{l}1 \\
0.920 \\
0.870 \\
0.947\end{array}$ & $\begin{array}{l}0.380 \\
0.381 \\
0.230\end{array}$ & $\begin{array}{l}2.228 \\
1.986 \\
3.896\end{array}$ \\
\hline Periods & $\begin{array}{l}1995-2004 \\
2005-2010 \\
>2010\end{array}$ & $\begin{array}{l}0.877 \\
0.129\end{array}$ & $\begin{array}{l}\mathrm{I} \\
\mathrm{I} .047 \\
0.648\end{array}$ & $\begin{array}{l}0.586 \\
0.369\end{array}$ & $\begin{array}{l}1.870 \\
1.135\end{array}$ \\
\hline Grade & $\begin{array}{l}1 \\
2 \\
3 \\
\text { Unknown }\end{array}$ & $\begin{array}{l}0.048 \\
0.980 \\
0.202\end{array}$ & $\begin{array}{l}1 \\
1.931 \\
1.008 \\
1.966\end{array}$ & $\begin{array}{l}1.005 \\
0.527 \\
0.695\end{array}$ & $\begin{array}{l}3.712 \\
1.928 \\
5.557 \\
\end{array}$ \\
\hline Size, $\mathrm{mm}$ & $\begin{array}{l}<20 \\
20-49.9 \\
\geq 50\end{array}$ & $\begin{array}{l}0.007 \\
<0.000 \text { I }\end{array}$ & $\begin{array}{l}1 \\
2.389 \\
18.02\end{array}$ & $\begin{array}{l}1.265 \\
9.189\end{array}$ & $\begin{array}{l}4.510 \\
35.34\end{array}$ \\
\hline
\end{tabular}

Abbreviation: DCIS, ductal carcinoma in situ; BC, breast cancer; NS, not significant.

Table 5 Factors associated with reoperation with mastectomy for invasive BC: multivariate analysis

\begin{tabular}{|c|c|c|c|c|c|}
\hline \multirow{2}{*}{\multicolumn{2}{|c|}{$\begin{array}{l}\text { Re-excision vs mastectomy } \\
\text { Invasive }\end{array}$}} & \multirow{3}{*}{\begin{tabular}{|l|}
$\boldsymbol{P}$-value \\
\\
$<0.0001$ \\
0.348
\end{tabular}} & \multirow{3}{*}{$\begin{array}{l}\text { OR } \\
1 \\
1.820 \\
1.175\end{array}$} & \multicolumn{2}{|l|}{$95 \% \mathrm{Cl}$} \\
\hline & & & & \multirow{2}{*}{$\begin{array}{l}\text { Inferior } \\
\\
1.369 \\
0.839\end{array}$} & \multirow{2}{*}{\begin{tabular}{|l} 
Superio \\
2.418 \\
1.646
\end{tabular}} \\
\hline Histology & $\begin{array}{l}\text { Ductal } \\
\text { Lobular } \\
\text { Others }\end{array}$ & & & & \\
\hline LVI & $\begin{array}{l}\text { No } \\
\text { Yes }\end{array}$ & 0.066 & $\begin{array}{l}1 \\
1.247\end{array}$ & 0.985 & 1.578 \\
\hline Periods & $\begin{array}{l}1995-2004 \\
2005-2010 \\
>2010\end{array}$ & $\begin{array}{l}0.552 \\
<0.0001\end{array}$ & $\begin{array}{l}1 \\
0.917 \\
0.429\end{array}$ & $\begin{array}{l}0.688 \\
0.328\end{array}$ & $\begin{array}{l}1.221 \\
0.562\end{array}$ \\
\hline Focality & $\begin{array}{l}\text { Unifocal } \\
\text { Bifocal } \\
\text { Multifocal }\end{array}$ & $\begin{array}{l}<0.0001 \\
<0.0001\end{array}$ & $\begin{array}{l}1 \\
2.955 \\
9.334\end{array}$ & $\begin{array}{l}2.161 \\
5.497\end{array}$ & $\begin{array}{l}4.041 \\
15.85\end{array}$ \\
\hline Grade & $\begin{array}{l}1 \\
2 \\
3\end{array}$ & $\begin{array}{l}0.024 \\
0.039\end{array}$ & $\begin{array}{l}1 \\
1.336 \\
1.393 \\
\end{array}$ & $\begin{array}{l}1.039 \\
1.016\end{array}$ & $\begin{array}{l}1.719 \\
1.909\end{array}$ \\
\hline DCIS component & DCIS - IT and peripheric & 0.393 & 0.911 & 0.736 & 1.128 \\
\hline Positive margins & $\begin{array}{l}\text { Focal } \\
\text { Diffuse }\end{array}$ & $<0.0001$ & $\begin{array}{l}1 \\
2.508\end{array}$ & 1.880 & 3.346 \\
\hline Age, years & $\begin{array}{l}\leq 40 \\
4 I-50 \\
5 I-74 \\
\geq 75\end{array}$ & $\begin{array}{l}0.835 \\
0.002 \\
0.309\end{array}$ & $\begin{array}{l}I \\
0.963 \\
0.602 \\
0.768\end{array}$ & $\begin{array}{l}0.673 \\
0.433 \\
0.462\end{array}$ & $\begin{array}{l}1.377 \\
0.836 \\
1.276\end{array}$ \\
\hline Global size, $\mathrm{mm}$ & $\begin{array}{l}<20 \\
20-49.9 \\
\geq 50\end{array}$ & $\begin{array}{l}<0.0001 \\
<0.0001\end{array}$ & $\begin{array}{l}I \\
2.372 \\
18.16 \\
\end{array}$ & $\begin{array}{l}1.856 \\
13.32\end{array}$ & $\begin{array}{l}3.032 \\
24.75\end{array}$ \\
\hline Phenotype & $\begin{array}{l}\text { ER+ Her2- } \\
\text { ER+ Her2+ } \\
\text { ER- Her2+ } \\
\text { ER- Her2- }\end{array}$ & $\begin{array}{l}0.048 \\
0.007 \\
0.20\end{array}$ & $\begin{array}{l}1 \\
1.453 \\
2.085 \\
1.294\end{array}$ & $\begin{array}{l}1.003 \\
1.228 \\
0.873\end{array}$ & $\begin{array}{l}2.105 \\
3.540 \\
1.919\end{array}$ \\
\hline
\end{tabular}

Abbreviations: $\mathrm{BC}$, breast cancer; LVI, lymphovascular peritumoral invasion; DCIS, ductal carcinoma in situ; IT, intratumoral. 
Table 6 Factors associated with third operation with mastectomy: multivariate analysis

\begin{tabular}{|c|c|c|c|c|c|}
\hline \multirow{2}{*}{\multicolumn{2}{|c|}{$\begin{array}{l}\text { Factors of mastectomy third operation } \\
\text { Among re-excision }\end{array}$}} & \multirow{3}{*}{\begin{tabular}{|l|}
$P$-value \\
\\
0.937 \\
0.622 \\
0.903 \\
\end{tabular}} & \multirow{3}{*}{\begin{tabular}{l|} 
OR \\
1 \\
1.033 \\
1.244 \\
1.060 \\
\end{tabular}} & \multicolumn{2}{|l|}{ 95\% Cl } \\
\hline & & & & \multirow{2}{*}{$\begin{array}{l}\text { Inferior } \\
\\
0.463 \\
0.523 \\
0.414\end{array}$} & \multirow{2}{*}{\begin{tabular}{|l} 
Superior \\
\\
2.305 \\
2.960 \\
2.717
\end{tabular}} \\
\hline Histology & $\begin{array}{l}\text { DCIS } \\
\text { Ductal } \\
\text { Lobular } \\
\text { Others }\end{array}$ & & & & \\
\hline Global size, $\mathrm{mm}$ & $\begin{array}{l}<20 \\
20-49.9 \\
\geq 50\end{array}$ & $\begin{array}{l}<0.0001 \\
<0.0001\end{array}$ & $\begin{array}{l}1 \\
2.374 \\
16.72\end{array}$ & $\begin{array}{l}1.463 \\
9.841\end{array}$ & $\begin{array}{l}3.852 \\
28.42\end{array}$ \\
\hline Focality & $\begin{array}{l}\text { Unifocal } \\
\text { Bifocal } \\
\text { Multifocal }\end{array}$ & $\begin{array}{l}0.205 \\
0.017\end{array}$ & $\begin{array}{l}1 \\
1.471 \\
3.120\end{array}$ & $\begin{array}{l}0.809 \\
1.225\end{array}$ & $\begin{array}{l}2.675 \\
7.948\end{array}$ \\
\hline LVI & $\begin{array}{l}\text { No } \\
\text { Yes }\end{array}$ & 0.532 & \begin{tabular}{|l|}
1 \\
0.868
\end{tabular} & 0.556 & 1.355 \\
\hline DCIS component & DCIS - IT and peripheric & 0.539 & 0.884 & 0.596 & $1.31 \mathrm{I}$ \\
\hline Phenotype & $\begin{array}{l}\text { ER+ Her2- } \\
\text { ER+ Her2+ } \\
\text { ER- Her2+ } \\
\text { ER- Her2- }\end{array}$ & $\begin{array}{l}0.175 \\
0.524 \\
0.787\end{array}$ & $\begin{array}{l}1 \\
1.503 \\
1.338 \\
0.896\end{array}$ & $\begin{array}{l}0.834 \\
0.546 \\
0.403\end{array}$ & $\begin{array}{l}2.709 \\
3.277 \\
1.989\end{array}$ \\
\hline Age, years & $\begin{array}{l}\leq 40 \\
4 \mathrm{I}-50 \\
5 \mathrm{I}-74 \\
\geq 75\end{array}$ & $\begin{array}{l}0.811 \\
0.005 \\
0.033\end{array}$ & \begin{tabular}{|l|}
$I$ \\
.073 \\
0.448 \\
0.281
\end{tabular} & $\begin{array}{l}0.601 \\
0.256 \\
0.087\end{array}$ & $\begin{array}{l}1.916 \\
0.784 \\
0.902\end{array}$ \\
\hline
\end{tabular}

Abbreviations: DCIS, ductal carcinoma in situ; LVI, lymphovascular peritumoral invasion; IT, intratumoral.

reported in 2016 that $2 \mathrm{~mm}$ margin minimizes the risk of ipsilateral breast tumor recurrence (IBTR) compared with smaller negative margins, using a meta-analysis of margin width and IBTR from a systematic review.

A conservative reoperation was done in $\sim 50 \%$ of patients with DCIS or ductal invasive BC and lesser for lobular invasive $\mathrm{BC}(\sim 30 \%)$ with a decrease in mastectomy rates among successive periods analyzed. In our study, interval times between first surgery and adjuvant treatments increased for patients who needed reoperation but without significant impact on survival.

A third intervention for mastectomy had been reported in three studies, ${ }^{1,3,6}$ and in our study: mastectomy rate after re-excision was $13.54 \%(1,059 / 7,818,95 \%$ CI $12.8-14.3)$. Mastectomy rates among 108,446 patients with reoperation in the literature review were $40.77 \%$ (95\% CI 40.5-41.1, range: $10.7-62.1)$ and $55.4 \%$ (95\% CI 54.6-56.2) for studies with analysis of a third intervention for mastectomy $(8,309 / 14,998) \cdot{ }^{1,3,6}$

When analysis of mastectomy rates was performed according to the three periods of treatment (DCIS and invasive), we observed a decrease in mastectomy rates among successive periods from $59.62 \%(2,739 / 4,594,95 \% \mathrm{CI}$ $58.2-61.0)$ to $48.8 \%(2,532 / 5,191,95 \%$ CI $47.4-50.2)$ and $36.81 \%(6,892 / 17,903,95 \%$ CI $36.1-37.5){ }^{3,4,6,7,9,11}$ In the study reported by Wilke et al, ${ }^{5}$ the mastectomy rate was $37.9 \%$ for patients operated between 2004 and 2010.

Mastectomy rates were different according to histological types of tumor: $49.7 \%(1,960 / 3,942,95 \%$ CI $48.1-51.3)$ for DCIS, $, 1,46,8,11,13,1450.9 \%(9,446 / 18,566,95 \%$ CI $50.2-51.6)$ for invasive BC, 55.7\% (95\% CI 54.5-56.9) for ductal invasive BC, $70.5 \%$ (95\% CI 68.3-72.7) for lobular invasive BC, and $60.9 \%$ (95\% CI 57.8-64.0) for other invasive histological types. ${ }^{2}$

After initial oncoplastic surgery, close or positive margins was reported in $12.3 \%(342 / 2,772,95 \%$ CI $11.1-13.5)$ of patients in a meta-analysis reported in $2014,{ }^{23}$ and the mastectomy rate for reoperation after initial oncoplastic resection was high: $64 \%$ in Clough et al's ${ }^{24}$ study and $61.3 \%$ in Losken et $\mathrm{al}^{23}$ meta-analysis.

Interval time between surgery or first surgery for patients who need reoperation and adjuvant treatment: even if interval time was higher for patients with reoperation, we did not observe impact on survival results between patients with or without reoperation.

\section{Conclusion}

A decrease of reoperation rate and mastectomy rate in future years is probably in relation with negative margin resection without several $\mathrm{mm}$ of margin, more accurate preoperative radiologic analysis, particularly for lobular and multifocal 
tumors; more oncoplastic resections; and more NAC. A third intervention with mastectomy was required in about $13 \%-14 \%$ of patients. Information about risk of a third intervention should be given to patients who require reoperation.

Even if interval time between surgery and adjuvant treatments was higher for patients with reoperation, survival results were not different between patients with and without reoperation.

\section{Ethics approval and consent to participate}

This work was approved by our institutional review board (IPC - Comité d'Orientation Stratégique). All procedures performed in this study involving human participants were done in accordance with the French ethical standards and 2008 Declaration of Helsinki. All included patients provided written informed consent before surgery, including for the use of their data for research.

\section{Author contributions}

All authors contributed equally to this study. All authors contributed toward data analysis, drafting and critically revising the paper, gave final approval of the version to be published, and agree to be accountable for all aspects of the work.

\section{Disclosure}

The authors report no conflicts of interest in this work.

\section{References}

1. van Leeuwen MT, Falster MO, Vajdic CM, et al. Reoperation after breast-conserving surgery for cancer in Australia: statewide cohort study of linked hospital data. BMJ Open. 2018;8(4):e020858.

2. Vos EL, Siesling S, Baaijens MHA, et al. Omitting re-excision for focally positive margins after breast-conserving surgery does not impair disease-free and overall survival. Breast Cancer Res Treat. 2017;164(1): 157-167.

3. Fisher S, Yasui Y, Dabbs K, Winget M. Re-excision and survival following breast conserving surgery in early stage breast cancer patients: a population-based study. BMC Health Serv Res. 2018;18(1):94.

4. Jeevan R, Cromwell DA, Trivella M, et al. Reoperation rates after breast conserving surgery for breast cancer among women in England: retrospective study of hospital episode statistics. BMJ. 2012;345: e4505.

5. Wilke LG, Czechura T, Wang C, et al. Repeat surgery after breast conservation for the treatment of stage 0 to II breast carcinoma: a report from the National Cancer data base, 2004-2010. JAMA Surg. 2014;149(12): 1296-1305.

6. Langhans L, Jensen MB, Talman MM, Vejborg I, Kroman N, Tvedskov TF. Reoperation rates in ductal carcinoma in situ vs invasive breast cancer after wire-guided breast-conserving surgery. JAMA Surg. 2017;152(4):378.
7. Landercasper J, Whitacre E, Degnim AC, Al-Hamadani M. Reasons for re-excision after lumpectomy for breast cancer: insight from the American Society of Breast Surgeons Mastery(SM) database. Ann Surg Oncol. 2014;21(10):3185-3191.

8. Romics L, Macaskill EJ, Fernandez T, et al. A population-based audit of surgical practice and outcomes of oncoplastic breast conservations in Scotland - An analysis of 589 patients. Eur J Surg Oncol. 2018;44(7):939-944.

9. Morrow M, Abrahamse P, Katz SJ. Trend analysis on reoperation after lumpectomy for breast cancer-reply. JAMA Oncol. 2018;4(5):747.

10. Morrow M, Jagsi R, Alderman AK, et al. Surgeon recommendations and receipt of mastectomy for treatment of breast cancer. JAMA. 2009;302(14):1551-1556.

11. Philpott A, Wong J, Elder K, Gorelik A, Mann GB, Skandarajah A. Factors influencing reoperation following breast-conserving surgery. ANZ J Surg. 2018;88(9):922-927.

12. Findlay-Shirras LJ, Outbih O, Muzyka CN, Galloway K, Hebbard PC, Nashed M. Predictors of residual disease after breast conservation surgery. Ann Surg Oncol. 2018;25(7):1936-1942.

13. Hughes L, Hamm J, Mcgahan C, Baliski C. Surgeon volume, patient age, and tumor-related factors influence the need for Re-Excision after breast-conserving surgery. Ann Surg Oncol. 2016;23(Suppl 5):656-664.

14. Heelan Gladden AA, Sams S, Gleisner A, et al. Re-excision rates after breast conserving surgery following the 2014 SSO-ASTRO guidelines. Am J Surg. 2017;214(6):1104-1109.

15. Tang SS, Kaptanis S, Haddow JB, et al. Current margin practice and effect on re-excision rates following the publication of the SSO-ASTRO consensus and ABS consensus guidelines: a national prospective study of 2858 women undergoing breast-conserving therapy in the UK and Ireland. Eur J Cancer. 2017;84:315-324.

16. Amabile MI, Mazouni C, Guimond C, et al. Factors predictive of reexcision after oncoplastic breast-conserving surgery. Anticancer Res. 2015;35(7):4229-4234.

17. Houvenaeghel G, Lambaudie E, Bannier M, et al. Re-operation and mastectomy rates after breast conservative surgery for positive or close margins: a review. Clin Surg. 2018;3:2149.

18. Chagpar AB, Killelea BK, Tsangaris TN, et al. A randomized, controlled trial of cavity shave margins in breast cancer. $N$ Engl J Med. 2015;373(6):503-510.

19. Pleijhuis RG, Graafland M, de Vries J, Bart J, de Jong JS, van Dam GM. Obtaining adequate surgical margins in breast-conserving therapy for patients with early-stage breast cancer: current modalities and future directions. Ann Surg Oncol. 2009;16(10):2717-2730.

20. Kuerer HM, Smith BD, Chavez-Macgregor M, et al. DCIS margins and breast conservation: MD Anderson cancer center multidisciplinary practice guidelines and outcomes. J Cancer. 2017;8(14):2653-2662.

21. van Zee KJ, Subhedar P, Olcese C, Patil S, Morrow M. Relationship between margin width and recurrence of ductal carcinoma in situ: analysis of 2996 women treated with breast-conserving surgery for 30 years. Ann Surg. 2015;262:623-631.

22. Morrow M, van Zee KJ, Solin LJ, et al. Society of surgical oncologyAmerican Society for Radiation Oncology-American Society of Clinical Oncology Consensus Guideline on margins for breast-conserving surgery with whole-breast irradiation in ductal carcinoma in situ. Ann Surg Oncol. 2016;23(12):3801-3810.

23. Losken A, Styblo TM, Carlson GW, Jones GE, Amerson BJ. Management algorithm and outcome evaluation of partial mastectomy defects treated using reduction or mastopexy techniques. Ann Plast Surg. 2007;59(3):235-242.

24. Clough KB, van La Parra RFD, Thygesen HH, et al. Long-term results after oncoplastic surgery for breast cancer: a 10-year follow-up. Ann Surg. 2018;268(1):165-171. 


\section{Publish your work in this journal}

Cancer Management and Research is an international, peer-reviewed open access journal focusing on cancer research and the optimal use of preventative and integrated treatment interventions to achieve improved outcomes, enhanced survival and quality of life for the cancer patient. The manuscript management system is completely online and includes a very quick and fair peer-review system, which is all easy to use. Visit http://www.dovepress.com/testimonials.php to read real quotes from published authors.

Submit your manuscript here: https://www.dovepress.com/cancer-management-and-research-journal 